\title{
An Adaptive Technique for Accurate Feature Extraction from Regular and Irregular Image Data
}

\author{
Sonya Coleman ${ }^{1}$, Shanmuglingam Suganthan ${ }^{2}$, and Bryan Scotney ${ }^{3}$ \\ ${ }^{1}$ University of Ulster, Magee, BT48 7JL, Northern Ireland \\ ${ }^{2}$ Smart Sensors Ltd, University of Bath Innovation Centre, Broad Quay, Bath, BA1 1UD \\ ${ }^{3}$ University of Ulster, Coleraine, BT52 1SA, Northern Ireland \\ SA.Coleman@Ulster.ac.uk, S.Suganthan@ieee.org, \\ BW.Scotney@Ulster.ac.uk
}

\begin{abstract}
We present a single multi-scale gradient-based feature extraction algorithm that can be directly applied to irregular or regular image data and hence can be used on both range and intensity images. We illustrate the accuracy of this approach using the Figure of Merit evaluation technique on real images, demonstrating that the application of this approach to both range and intensity images is more accurate than the equivalent approach of applying a gradient operator, such as Sobel, to an intensity image and, separately, the scan-line approximation approach to range images.
\end{abstract}

Keywords: Range Data, Gradient Operator, Feature Extraction.

\section{Introduction}

For many years, feature extraction techniques have been developed for use on intensity images, the earliest being the simple gradient operators of Sobel and Prewitt, and extensive efforts have been made to develop multi-scale feature extraction approaches, for example [8, 17]. Recently computer vision applications have increasingly begun to use range image data $[11,12]$ instead of, or in conjunction with, intensity image data [7]. This is largely because range imagery can be used to obtain reliable descriptions of 3-D scenes; a range image contains distance measurements from a selected reference point or plane to surface points of objects within a scene [4], allowing more information about the scenes to be recovered [3]. However, techniques that can be used for feature extraction directly from more than one image type and in particular that can be directly applied to both range and intensity images are not readily available $[2,9]$. Typically approaches for feature extraction in intensity or range images can only be applied principally to that specific image type; none of the typical approaches can be readily applied to both image types without image preprocessing. As much research has been undertaken in trying to integrate $2 \mathrm{D}$ and $3 \mathrm{D}$ images [2], specifically in application areas such as medical imaging [6] and face recognition $[5,21]$, we believe that a single approach that can be used on both image types would greatly aid the process of image fusion.

When using range images the problem of data irregularity may be encountered [13, 14]. In $[15,16]$ we addressed the data distribution problem by generating directional 
derivate operators that are shape adaptive, and hence can be applied directly to both irregularly and regularly distributed data, thus providing the facility for the operators to be applied directly to both range and intensity images. In $[15,16]$ we noted that the standard thresholding technique for finding edges, typically applied to intensity images, is not appropriate for use on range image data, as it does not identify edges, but surfaces in range images; to overcome this drawback, we identified a process of finding significant changes in the gradient response as a means of determining edges in range images.

In [16] we present multi-scale gradient operators for direct use on range images containing either irregularly or regularly distributed image data, designed within the finite element framework. In this paper we demonstrate how the flexibility of this approach enables us to apply our multi-scale technique to either range or intensity images, and the performance evaluation illustrates the accuracy of this approach. Section 2 provides a brief overview of the multi-scale algorithm published in [16] and discusses how such an approach can be readily applied to both intensity and range images. Section 3 provides initial Figure of Merit results for the approach, demonstrating that our single approach works better than the equivalent use of two separate feature extraction algorithms, and Section 4 provides a summary and details of future work.

\section{Overview of Adaptive Bilinear-Gaussian (BG) Operators}

In order to address the use of either intensity or range images, we consider an image to be represented by a spatially irregular sample of values of a continuous function $u(x, y)$ of depth value on a domain $\Omega$. Our operator design is then based on the use of a quadrilateral mesh in which the nodes are the sample points. With each node $i$ in the mesh is associated a piecewise bilinear basis function $\phi_{i}(x, y)$ which has the properties $\phi_{i}\left(x_{j}, y_{j}\right)=1$ if $i=j$ and $\phi_{i}\left(x_{j}, y_{j}\right)=0$ if $i \neq j$, where $\left(x_{j}, y_{j}\right)$ are the coordinates of the nodal point $j$ in the mesh. Thus $\phi_{i}(x, y)$ is a "tent-shaped" function with support restricted to a small neighbourhood centred on node $i$ consisting of only those elements that have node $i$ as a vertex. The image function $u$ is approximately represented by a function

$$
U(x, y)=\sum_{j=1}^{N} U_{j} \phi_{j}(x, y)
$$

in which the parameters $\left\{U_{1}, \ldots, U_{N}\right\}$ are mapped from the image pixel values (either intensity or range) at the $N$ irregularly located nodal points.

The complete operator design is described in detail in [16] whereby we formulate image operators that correspond to weak forms in the finite element method; for example, corresponding to a first directional derivative $\partial u / \partial b \equiv \underline{b} \cdot \underline{\nabla} u$ we may use a test function $v \in H^{1}(\Omega)$ to define the weak form

$$
E(u)=\int_{\Omega} \underline{b} \cdot \underline{\nabla} u v d \Omega,
$$


where $\underline{b}=(\cos \theta, \sin \theta)$ is the unit direction vector. Since we are focussing on multiscale operators that can explicitly embrace the concept of size and shape variability, the design procedure in [16] uses a finite-dimensional test space $T_{\sigma}^{h} \subset H^{1}$ that explicitly embodies a size parameter $\sigma$ that is determined by the local data distribution. Using such test functions, the first order functional is defined as

$$
E_{i}^{\sigma}(U)=\int_{\Omega_{i}^{\sigma}} \underline{b}_{i} \cdot \underline{\nabla} U \psi_{i}^{\sigma} d \Omega_{i},
$$

where sets of test functions $\psi_{i}^{\sigma}(x, y), i=1, \ldots, N$, are used when defining irregular derivative-based operators and the chosen test function is a Gaussian basis function. Hence we refer to our technique as the use of Bilinear-Gaussian operators. Further details of operator construction can be found in [16].

Figure 1 illustrates a local " $3 \times 3$ " neighbourhood on an irregular mesh. When using range image data, within each neighbourhood a different size parameter is computed for each quadrant of the neighbourhood, enabling the Gaussian test function to adapt to the local area more accurately. However, when using an intensity image, the data distribution will be regular and therefore $W_{\sigma}^{e_{1}}=W_{\sigma}^{e_{2}}=W_{\sigma}^{e_{3}}=W_{\sigma}^{e_{4}}$. As illustrated in Figure 1, $W_{\sigma}^{e_{m}}$ is chosen as the "diagonal" of the neighbourhood from the operator centre $\left(x_{i}, y_{i}\right)$, and in each case the quadrant scale parameter $\sigma_{m}=W_{\sigma}^{e_{m}} / 1.96$ ensures that the diagonal of the quadrant through $\left(x_{i}, y_{i}\right)$ encompasses $95 \%$ of the cross-section of the Gaussian.

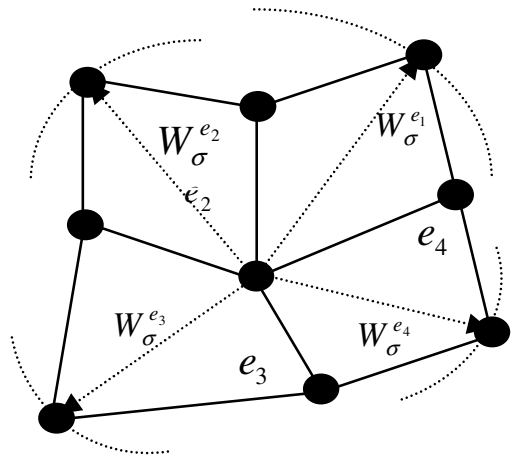

Fig. 1. Local $3 \times 3$ operator neighbourhood

In the case where intensity images are used, the $3 \times 3$ (or any other sized) operator needs to be computed only once and applied appropriately across the image plane; however, as suggested by the change in $W_{\sigma}^{e_{m}}$ within any neighbourhood, construction of the operators on an irregular quadrilateral grid, and hence when using range image data, differs in that it is no longer appropriate to build an entire operator, as each operator throughout an irregular mesh will differ with respect to the operator neighbourhood shape. When using an irregular grid with a range image, we work on 
an element-by-element basis, taking advantage of the flexibility offered by the finite element method as a means of creating irregular operators to encompass the data available in any local neighbourhood.

\section{Results}

In order to obtain features after application of our technique to range and intensity images, two different methods of thresholding must be used. In the case of using intensity images, features are found using the standard thresholding approach. However, when gradient operators are applied to range images, standard thresholding yields object surfaces rather than edges; hence we must look for significant changes in the gradient responses of the range image.

For evaluation purposes, we use the well-known Figure of Merit (FoM) measure [1] and compare our proposed technique (further denoted as BG) with that of the well-known scan-line approximation algorithm [10] (further denoted as SA). Pratt [1] considered three major areas of error associated with the determination of an edge: missing valid edge points; failure to localise edge points; and classification of noise fluctuations as edge points. Pratt therefore introduced the FoM technique as one that balances these three types of error, defined as

$$
R=\frac{1}{\max \left(I_{A}, I_{I}\right)} \sum_{i=1}^{I_{A}} \frac{1}{1+\alpha d^{2}}
$$

Here $I_{A}$ is the actual number of edge pixels detected, $I_{I}$ is the ideal number of edge pixels, $d$ is the separation distance of a detected edge point normal to a line of ideal edge points, and $\alpha$ is a scaling factor, most commonly chosen to be $1 / 9$, although this value may be adjusted to penalize edges that are localized but offset from the true edge position. Initially we evaluated the gradient operators with respect to their use

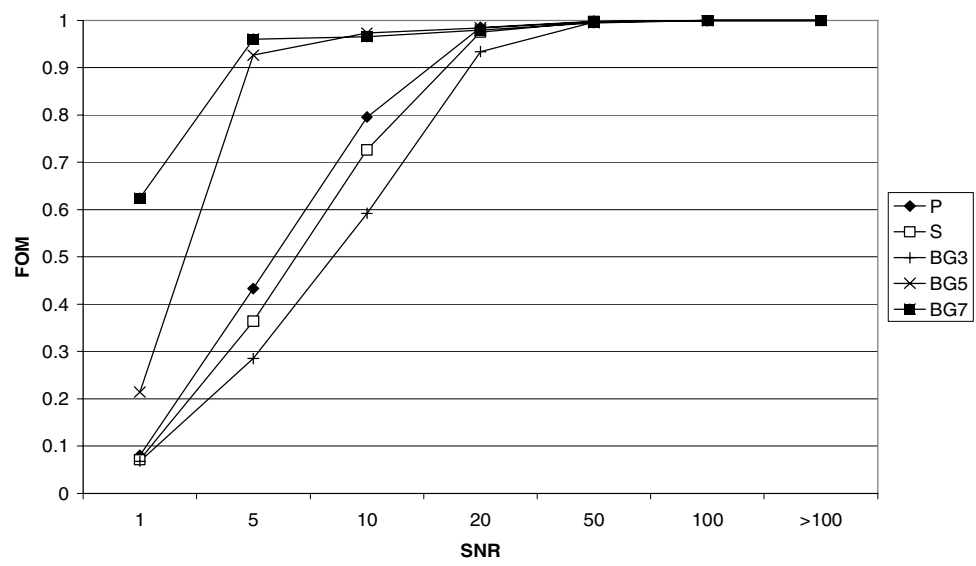

Fig. 2. FoM values vs SNR for operators on a vertical edge 
on intensity images. Figure 2 presents comparative results on a vertical ramp edge using the Prewitt and Sobel operators (denoted $\mathrm{P}$ and $\mathrm{S}$ ) and the proposed $3 \times 3,5 \times 5$ and $7 \times 7$ Bilinear-Gaussian gradient operators (denoted as BG3, BG5 and BG7). The results illustrate that the multi-scale approach works well on intensity images when compared with the well-known operators of Prewitt and Sobel.

In order to evaluate the accuracy of using just one approach for feature extraction on both intensity and range images, we initially apply the operators to the intensity image (Figure 3(a)) and select the visually best edge map for each operator, an example of which is illustrated in Figure 4.

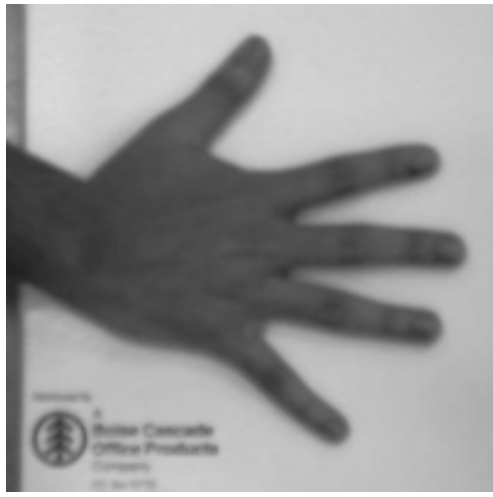

(a) Intensity image

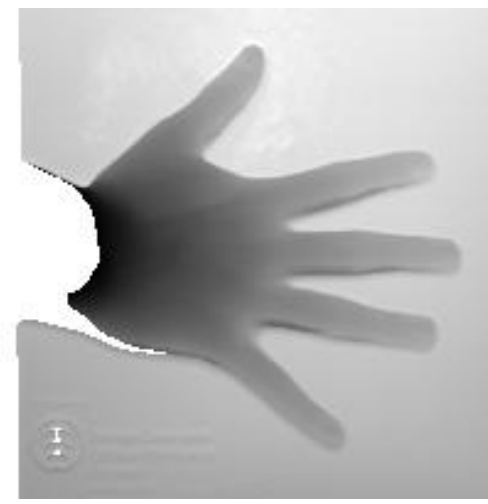

(b) Range image

Fig. 3. Original images

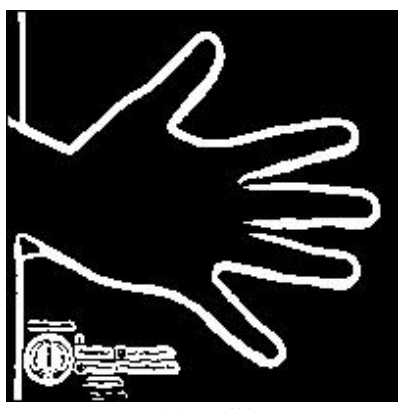

(a) BG3

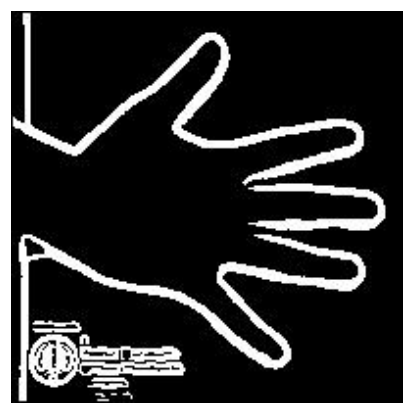

(b) Sobel

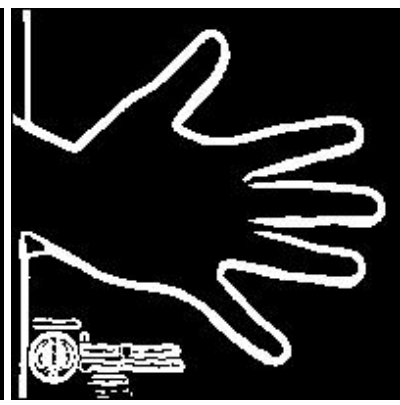

(c) Prewitt

Fig. 4. Example of edge maps (ground truth) using the intensity image in Figure 3(a)

We then run a series of experiments whereby we apply each technique, $3 \times 3,5 \times 5$ and $7 \times 7$ Bilinear-Gaussian gradient operators (denoted as BG3, BG5 and BG7) and the scan-line approach of [10], to the corresponding range image (Figure 3(b)) over a range of practical thresholds. Using each intensity image feature map as a possible ground truth match, we compute the FoM value at each threshold for every operator 

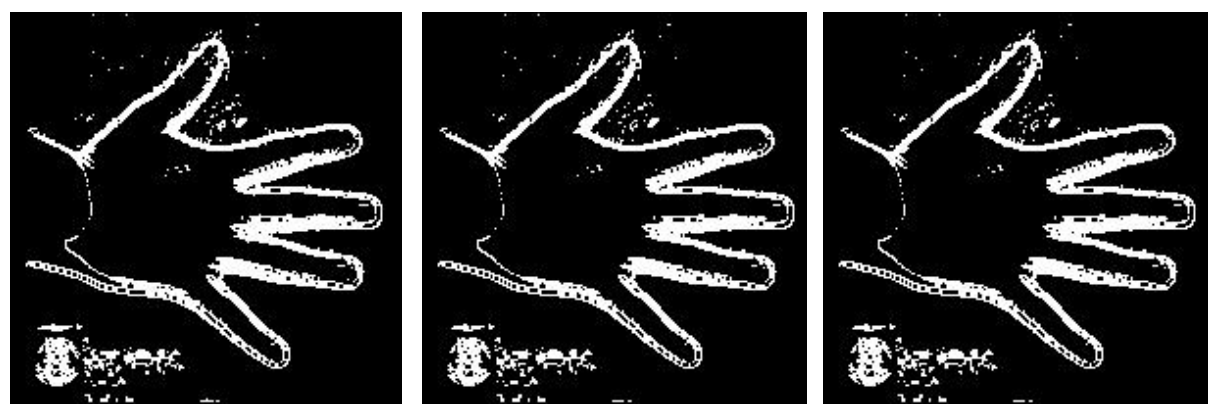

(a) Range edge map from (b) Range edge map from (c) Range edge map from BG3, providing best FoM BG3, providing best FoM BG3, providing best FoM using BG3 intensity edge map using Sobel intensity edge map using Prewitt intensity edge as ground truth as ground truth map as ground truth
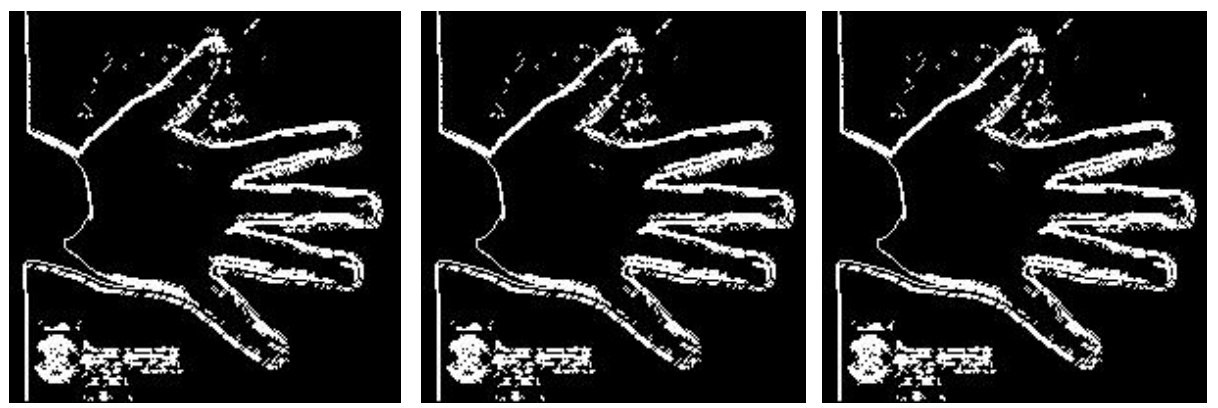

(d) Range edge map from SA, (e) Range edge map from SA, (f) Range edge map from SA, providing best FoM using providing best FoM using providing best FoM using BG3 intensity edge map as Sobel intensity edge map as Prewitt intensity edge map as ground truth ground truth ground truth

Fig. 5. Edge maps corresponding to maximum FoM value, using the ground truth in Figure 4: (a)-(c)BG3 operator; (d)-(f) SA approach [10]

in order to determine the range image feature map than provides the best FoM value and hence the best match between intensity and range edge maps; an example of such optimal edge maps are presented in Figure 5.

It should be noted in the Figure 5 that the edge maps generated using the scan-line approach that provide the best FoM value are relatively noisy in comparison to the corresponding edge maps using the BG3 operator. It's also worth noting that our technique automatically finds all features, whereas the approach in [10] does not automatically find the object boundary via the scan line approximation but instead assumes the boundary at the transition between data and no data in the range image.

Table 1 provides the maximum computed FoM values when each range image feature map is compared to the corresponding ground truth intensity image. We can see that in each case the proposed adaptive Bilinear-Gaussian approach provides a higher FoM value than the scan-line approach. Moreover, and the main focus of the paper, using a single operator (BG3) on both the intensity image and the range image 
Table 1. Computed FoM values and \% match values

\begin{tabular}{|c|c|c|c|}
\hline $\begin{array}{l}\text { Operator used for } \\
\text { ground truth on } \\
\text { intensity image }\end{array}$ & & $\begin{array}{c}\text { New Bilinear- } \\
\text { Gaussian technique } \\
\text { (BG) }\end{array}$ & $\begin{array}{c}\text { Scan line } \\
\text { approach } \\
\text { (SA) }\end{array}$ \\
\hline \multirow[t]{4}{*}{ BG3 } & FoM & 0.8229 & 0.7953 \\
\hline & Total pixel match & $90.07 \%$ & $89.39 \%$ \\
\hline & Edge pixel match & $61.99 \%$ & $59.38 \%$ \\
\hline & Background pixel match & $94.30 \%$ & $93.91 \%$ \\
\hline \multirow[t]{4}{*}{ BG5 } & FoM & 0.8223 & 0.8022 \\
\hline & Total pixel match & $88.67 \%$ & $88.71 \%$ \\
\hline & Edge pixel match & $62.02 \%$ & $62.02 \%$ \\
\hline & Background pixel match & $93.36 \%$ & $93.40 \%$ \\
\hline \multirow[t]{4}{*}{ BG7 } & FoM & 0.8201 & 0.7789 \\
\hline & Total pixel match & $88.01 \%$ & $87.74 \%$ \\
\hline & Edge pixel match & $64.16 \%$ & $62.93 \%$ \\
\hline & Background pixel match & $92.78 \%$ & $92.70 \%$ \\
\hline \multirow[t]{4}{*}{ Sobel } & FoM & 0.8056 & 0.7965 \\
\hline & Total pixel match & $90.14 \%$ & $89.41 \%$ \\
\hline & Edge pixel match & $60.89 \%$ & $59.41 \%$ \\
\hline & Background pixel match & $94.57 \%$ & $93.96 \%$ \\
\hline \multirow[t]{4}{*}{ Prewitt } & FoM & 0.7977 & 0.7965 \\
\hline & Total pixel match & $90.11 \%$ & $89.34 \%$ \\
\hline & Edge pixel match & $60.62 \%$ & $61.06 \%$ \\
\hline & Background pixel match & $94.64 \%$ & $93.68 \%$ \\
\hline
\end{tabular}

provides a higher FoM value than the equivalent individual use of either Sobel or Prewitt on the intensity image followed by using BG3 on the range image. Table 1 also provides details on overall pixel matching in the edge maps for both edge and background pixels.

\section{Summary}

With the increase in imaging modalities available, image matching and image fusion have become prominent issues in recent years. We have demonstrated, via our evaluation, that the multi-scale technique presented in [16] for feature extraction on range images can be accurately used for feature extraction on both intensity and range images, regularly and irregularly distributed. The performance evaluation demonstrates that use of the proposed technique for edge detection on range and intensity images performs better than the combination of two different detection algorithms: one suitable for range images and one suitable for intensity images. We have thus provided a one-step approach to processing both image types, removing the need for application of two individual techniques. 


\section{Acknowledgment}

This work was supported by the U.K Research Council EPSRC under grant number EP/C006283/1. We would like to thank Professor Horst Bunke for providing us with the code for the scan line approximation algorithm in [10].

\section{References}

1. Abdou, I.E., Pratt, W.K.: Quantitative Design and Evaluation of Enhancement/ Threshold Edge Detectors. Proc. of IEEE 67(5) (May 1979)

2. Alshawabkeh, Y., Haala, N., Fritsch, D.: 2D-3D Feature Extraction and Registration of Real World Scenes. In: ISPRS Commission V Symposium Image Engineering and Vision Metrology, pp. 32-37 (2006)

3. Bellon, O., Silva, L.: New Improvements on Range Image Segmentation by Edge Detection Techniques. In: Proc. of Workshop on Artificial Intelligence and Computer Vision (November 2000)

4. Besl, P.J.: Active, optical range imaging sensors. Machine Vision and Apps. 1, 127-152 (1988)

5. Colombo, A., Cusano, C., Schettini, R.: Face3 a 2D+3D Robust Face Recognition System. In: Proc. of IEEE Int. Conf. on Image Analysis and Processing, pp. 393-398 (2007)

6. Cyr, C.M., Kamal, A.F., Sebastian, T.B., Kimia, B.B.: 2D-3D Registration Based on Shape Matching. In: IEEE Workshop Mathematical Methods in Biomedical Image Analysis, pp. 198-203 (2000)

7. Dias, P., et al.: Combining Intensity and Range Images for 3D Modelling. Proc. of the IEEE Int. Conf. on Image Processing (2003)

8. Ferreira, M., Kiranyaz, S., Gabbouj, M.: Multi-Scale Edge Detection and Object Extraction for Image Retrieval. In: Proc IEEE Int. Conf. on Acoustics, Speech and Signal Processing, vol. 2 (2006)

9. Gachter, S.: Results on Range Image Segmentation for Service Robots. Technical Report, Switerland

10. Jiang, X.Y., Bunke, H.: Fast Segmentation of Range Images into Planar Regions by Scan Line Grouping. Machine Vision and Applications 7(2), 115-122 (1994)

11. Katsoulas, D., Werber, A.: Edge Detection in Range Images of Piled Box-like Objects. In: Proc. Int. Conf. on Pattern Recognition pp.80-84 (2004)

12. Marshall, D., Lukas, G., Martin, R.: Robust Segmentation of Primitives from Range Data in the Presence of Geometric Degeneracy. IEEE Trans. PAMI 23(3), 304-314 (2001)

13. Maas, H.-G.: Planimetric and height accuracy of airborne laserscanner data - User requirements and system performance. In: Fritsch, D. (ed.) Proceedings of Photogrammetric Week, vol. 49. Wichmann Verlag (2003)

14. Lee, B.K., Yu, K., Pyeon, M.: Effective Reduction of Horzontal Error in Laser Scanning Information by Strip-Wise Least Squares Adjustments. ETRI Journal 25(2), 109-120 (2003)

15. Suganthan, S., Coleman, S.A., Scotney, B.W.: Range Image Feature Extraction with Varying Degrees of Data Irregularity. In: International Machine Vision and Image Processing Conference, pp. 33-40 (2007) 
16. Suganthan, S., Coleman, S.A., Scotney, B.W.: Scalable Operators for Feature Extraction on 3-D Data. Springer Tracts in Advanced Robotics 44, 263-272 (2008)

17. Tremblais, B., Augereau, B.: A fast multi-scale edge detection algorithm. Pattern Recognition Letters 25(6), 603-618 (2004)

18. Tsalakanidou, F., Malassiotis, S., Strintzis, M.G.: Integration of 2D and 3D Images for Enhanced Face Authentication. In: Proc. 6th IEEE Int. Conf. Automatic Face and Gesture Recognition (2004) 